\title{
Encoprese e intervenção psicológica: revisão de literatura
}

\section{Encopresis and psychological intervention: literature review}

\section{La encopresis e intervención psicológica: revisión de la literatura}

\section{Jéssica Aline Rovaris*}

Universidade Estadual Paulista - UNESP, Bauru, São Paulo, Brasil

\author{
Bárbara Trevizan Guerra** \\ Universidade Estadual Paulista - UNESP, Bauru, São Paulo, Brasil
}

\author{
Sandra Leal Calais*** \\ Universidade Estadual Paulista - UNESP, Bauru, São Paulo, Brasil
}

\section{Carmen Maria Bueno Neme****}

Universidade Estadual Paulista - UNESP, Bauru, São Paulo, Brasil

\begin{abstract}
RESUMO
Estudos têm demonstrado que a encoprese, transtorno relacionado ao controle esfincteriano, pode trazer impactos significativos para a vida do indivíduo, tais como baixa autoestima e déficit em interação social. Apesar de reconhecido o importante comprometimento que tal transtorno pode causar no desenvolvimento infantil, a literatura científica tem dado pouca importância ao tema, apresentando um baixo número de publicações sobre o assunto. Dessa forma, este artigo investigou as publicações nacionais em psicologia relacionadas à encoprese entre os anos de 1994 a 2013 nas bases de dados Lilacs e Scielo. Foram encontrados 231 artigos em toda a publicação para este assunto e período, e selecionados cinco para a análise, sendo aqueles que relatavam intervenção em encoprese. Desses artigos, quatro foram publicados na mesma revista e descreveram intervenções comportamentais, sendo três do mesmo autor. O quinto artigo era da abordagem psicanalítica. As pesquisas foram realizadas com crianças e adolescentes e as intervenções comportamentais tiveram êxito com remissão dos sintomas encopréticos. Os dados afirmam a deficiência em publicações na área e número restrito de pesquisadores investigando 0 tema, que é de relevância significativa.
\end{abstract}

Palavras-chave: encoprese, intervenção, desenvolvimento infantil.

\section{ABSTRACT}

Studies have shown that encopresis, related to bowel control disorder, can bring significant impact for the individual life, such as low self-esteem and deficits in social interaction. Despite the significant impairment recognized 
that this disorder can have on child development, the scientific literature has given little attention to the theme, with a low number of publications on the subject. Thus, this paper investigated Brazilian publications in psychology related to encopresis between 1994 and 2013 in data base Lilacs and Scielo. 231 articles were found and were selected those that reported only intervention in encopresis. There were five articles and four published by the same journal and described behavioral interventions, and one author wrote three. The other one was psychoanalytic. The surveys were conducted with children and adolescents and behavioral interventions have been successful with remission of encopresis symptoms. The data affirm the deficiency in publications in the area and possibly limited number of psychologists investigating the issue, which is of significant importance.

Keywords: encopresis, intervention, child development.

\begin{abstract}
RESUMEN
Los estudios han demostrado que la encopresis, relacionado con el trastorno intestinal control, pueden aportar importantes para la vida del individuo, tales como la baja autoestima y déficit en los efectos de interacción social. A pesar del deterioro significativo reconocido que este trastorno puede tener en el desarrollo del niño, la literatura científica ha prestado poca atención al tema, con un bajo número de publicaciones sobre el sujeto. Por lo tanto, este trabajo investiga las publicaciones brasileñas en psicología relacionada con encopresis entre los años 1994 y 2013 en las basis Lilacs e Scielo. Se encontraron 231 artículos e seleccionaron solamente artículos que informaron la intervención in encopresis. Se encontraron cinco artículos, y cuatro en la misma revista con intervenciones conductuales, siendo tres de lo mismo autor. Lo quinto articulo era de la psicoanálisis. Las encuestas se llevaron a cabo con las personas y las intervenciones conductuales han tenido éxito con la remisión de los síntomas encopréticos. Los datos confirman la deficiencia en las publicaciones de la zona y posiblemente limitado número de psicólogos que investiga el asunto, que es de gran importancia.
\end{abstract}

Palabras-clave: encopresis, intervención, desarrollo infantil.

\title{
1 Introdução
}

A encoprese, transtorno relacionado ao controle esfincteriano, pode trazer impactos significativos para a vida do indivíduo, como a baixa autoestima e dificuldades na socialização (Silvares \& Souza, 2001). Apesar da encoprese não acometer apenas crianças, estudos demonstram a estreita relação entre a encoprese e a aprendizagem do controle esfincteriano na infância (Blum, Taubman \& Nemeth, 2004; Bragado, 1998; Coehlo, 2011; Coelho, 2008; Silvares \& Souza, 2001). Segundo Wendy e William (2006), cerca de $4,1 \%$ das crianças entre 5 a 6 anos e $1,6 \%$ das crianças entre 11 e 12 anos apresentam encoprese.

O controle dos esfíncteres é adquirido, para a maior parte das crianças, de modo natural, por volta dos três anos, geralmente acompanhado pelo auxílio e supervisão dos cuidadores, processo denominado de treino ao toalete ou desfralde (Silvares \& Souza, 
2001). Dada a importância psicossocial do treino ao toalete para a criança, é importante que a família considere aspectos do desenvolvimento infantil ao iniciar o desfralde (Silvares \& Souza, 2001). Na infância, o comportamento de defecar é irregular e mais frequente do que nos adultos. Todavia, com o desenvolvimento, esses comportamentos diminuem junto com a alteração do padrão irregular das fezes (tamanho e consistência), o que possibilita para a criança maior controle do comportamento de defecar. Autores como Bragado (1998) e Silvares e Souza (2001) discutem que aspectos como o nível do desenvolvimento neuromotor e a maior lentidão dos meninos no processo natural de obtenção do controle dos esfíncteres precisam ser considerados pela família, pois, o início precoce e rígido no treino ao toalete pode dificultar e tornar sofrível esse processo, 0 que pode ser considerado fator de risco para a encoprese.

A Associação de Psiquiatria Americana (APA, 2002) descreve a encoprese como defecações e/ou evacuações realizadas em lugares impróprios, de forma voluntária ou não, em crianças que apresentem idade igual ou maior a quatro anos, ou desenvolvimento aproximado desta faixa etária. Além disso, é necessário que tal comportamento seja apresentado no mínimo uma vez por mês, por pelo menos três meses, e não pode estar relacionado a fatores fisiológicos. A deficiência no controle esfincteriano associada à encoprese é decorrente da perda da sensibilidade dos estímulos orgânicos que sinalizam a necessidade de defecação, pois a pessoa com encoprese passa a ignorar estes estímulos e reter as fezes (Coehlo, 2011). Em relação às classificações, a encoprese diferencia-se em (1) noturna ou diurna; (2) primária - casos em que nunca houve controle de esfíncter, ou secundária - o controle adquirido anteriormente; e, (3) retentiva - há retenção das fezes, ou não retentiva - existe um padrão diarreico (Coehlo, 2011; Cunningham \& Banez, 2006; Pinheiro \& Moreno, 1983). Segundo Coehlo (2011), a encoprese também está relacionada a outros comportamentos, tais como esconder roupas íntimas sujas, produzir fezes grandes e duras a cada três ou sete dias (invés de ir ao banheiro diariamente), entupir o vaso sanitário após a evacuação, manchar as roupas com fezes, cheirar a fezes, apresentar - abdômen distendido ou inchado, possuir infecções urinárias frequentes, apresentar enurese e ter poucos amigos.

Silvares e Souza (2001) apontam que pais ou cuidadores utilizam o treino ao toalete ou desfralde como auxílio no desenvolvimento do controle esfincteriano pelas crianças. Entretanto, a família, às vezes, pode apresentar poucas habilidades para a realização dessa tarefa. Por isso, é importante a compreensão de que o comportamento de usar o toalete de forma adequada envolve uma ampla e complexa cadeia de respostas, que se inicia desde o momento em que surge a vontade de usar o sanitário até a higienização completa das mãos. Em relação ao momento em que o treino deve ser iniciado, não há 
consenso quanto à faixa etária apropriada. Por exemplo, enquanto para Silvares e Souza (2001) é comum que o treino seja iniciado por volta dos três anos de idade, Brazelton (1962) afirma que o início do treino ao sanitário deve começar precocemente, aos dois anos. $\mathrm{Na}$ literatura científica internacional não se encontram atualizações sobre o período, mas em sites não científicos internacionais, também se comenta sobre o início aos dois anos.

As publicações nacionais, objeto deste estudo, referentes ao início do treino ao toalete são escassas, e não apresentam orientações sobre como lidar com os casos em que as crianças se recusam e/ou demonstram dificuldades nessa aprendizagem (Coelho, 2008). Para Blum et al. (2004), essa recusa pode se relacionar com prévias experiências de constipação, dificuldades ou dores na defecação. Mas, segundo Fleisher (2004), embora a constipação possa ser um elemento presente na recusa ao treino, nem todas as crianças que apresentam defecação dolorosa se recusam ao treinamento e, de forma contrária, nem todas as crianças com aversão ao treino ao banheiro tiveram experiências dolorosas. Além do treino ao toalete, é preciso considerar outros fatores de risco relacionados ao desenvolvimento da encoprese, tais como (a) prisão de ventre durante a infância, (b) baixo tônus muscular e falta de coordenação motora, (c) retardo da mobilidade intestinal, (d) pertencer ao sexo masculino, (e) abuso sexual, (f) dieta rica em gordura, pobre em fibras, e pouca ingestão de água, ( $\mathrm{g}$ ) ausência da prática de exercícios físicos, (h) recusa em usar o banheiro (em especial banheiros públicos), (i) atraso cognitivo, (j) dificuldade para identificar comportamentos fisiológicos que antecipam a evacuação, (k) negligência e (I) possuir vida desorganizada (Coehlo, 2011; Cox, Sutphen, Ling, Quillian, \& Borowitz, 1996). Quanto ao fator de risco de pertencer ao sexo masculino, pesquisa realizada por Wendy e William (2006) apontaram que a incidência do transtorno é três vezes mais comum em meninos.

Bragado (1998) afirma que há a ocorrência de múltiplos fatores no desenvolvimento da encoprese, assim como para a maioria dos transtornos infantis, dificultando a identificação nítida da etiologia primária do problema. Por fim, há ainda de se considerar os aspectos fisiológicos associados à constipação, às anomalias dietéticas, aos problemas do desenvolvimento, à predisposição e à aprendizagem relacionada ao treino inadequado ao toalete e controle intestinal.

A fim de que o treino ao toalete não se torne um evento aversivo para a criança, é importante observar o desenvolvimento dos comportamentos chamados de habilidades de prontidão que sinalizarão o sucesso, ou não, no uso adequado ao banheiro, por exemplo: expressar verbalmente a necessidade de evacuação; habilidades motoras como puxar ou abaixar as calças e levantar-se do vaso sanitário; compreender e seguir regras simples; e reter a 
urina por aproximadamente duas horas (o que indica certo controle de esfíncter) (Coehlo, 2011). Em relação ao tratamento, observou-se que a maioria utiliza estratégias isoladas. Entretanto, estratégias terapêuticas combinadas apresentam resultados mais satisfatórios, uma vez que tal patologia é multideterminada (Coehlo, 2011; Neves \& Calais, 2012; Silvares, 2001).

Pesquisas relatam que a medicação (Morais \& Fernandes, 2011), a terapia cognitiva comportamental (Knell, 1995) e ludoterapia comportamental (Gomes, 1998; Silvares, 2001) são formas eficazes para o tratamento da encoprese (Brazzelli \& Griffiths, 2006). Há ainda o treino comportamental ao toalete que representa uma técnica relevante, uma vez que promove aprendizagens, ou mudanças de comportamentos no reconhecimento dos estímulos direcionados à evacuação - aumento da sensibilidade do organismo aos estímulos que antecedem a evacuação. Exemplos dessas técnicas são permanecer sentado no vaso sanitário por cerca de 10 a 20 minutos após o café da amanhã e 20 minutos após o jantar, a fim de promover a percepção de que o reto está cheio. Sugere-se também urinar a cada duas horas, interrompendo o fluxo de duas a três vezes - a fim de fortalecer o músculo pélvico e melhorar o controle esfincteriano. Outro fator importante no treino consiste em reforçar positivamente pequenos ganhos comportamentais da criança tais como ficar no banheiro e permanecer limpa durante a evacuação, é importante ressaltar que punir o comportamento de encoprese pode aumentar o problema (Coehlo, 2011).

Dessa forma, segundo Coehlo (2011), a melhor estratégia é auxiliar a criança na compreensão do que está acontecendo por meio de histórias e elementos lúdicos. Ensiná-la a usar o banheiro regularmente por meio de um calendário, por exemplo, regular a alimentação e incentivar a prática de exercícios físicos também são estratégias relevantes presentes no tratamento. Mesmo utilizando o tratamento apropriado, estima-se que a criança precise de pelo menos seis meses a um ano para aprender o controle intestinal e eliminar os comportamentos encopréticos. Nesse tempo há a possibilidade de recaídas, e por isso é importante uma parceria bem estabelecida entre pais e profissionais da saúde, a fim de que a família consiga lidar com problemas secundários como raiva e frustração e ajude a criança a manter contato social e livrar-se de provocações por parte de colegas e irmãos. Além das recaídas, discute-se que mesmo após o tratamento cerca de $30 \%$ das crianças continuarão com constipação e terão alguns sintomas da encoprese na idade adulta. Assim, ao propor intervenções, deve-se atentar para diversos aspectos, como a dieta, a relação familiar, reeducação intestinal e os tratamentos medicamentosos.

Apesar da encoprese ser um transtorno que pode causar diversos danos fisiológicos e psicológicos na infância, podendo se estender até 
a fase adulta, menos de $40 \%$ das famílias com crianças com encoprese procuram por tratamento (Coehlo, 2011). Esse dado aponta para a necessidade de se aumentar o número de estudos sobre o assunto, a fim de viabilizar para a população em geral informações sobre o transtorno, aumentando, assim, a probabilidade da procura pelo tratamento. Faz-se relevante também investigar e analisar formas mais eficazes de tratamento (Coehlo, 2011).

Diante da escassez de produções bibliográficas brasileiras sobre o tema, da baixa procura por tratamento e das diferenças culturais relacionadas aos comportamentos parentais e infantis nessa fase do desenvolvimento da criança (Coehlo, 2011), das consequências nefastas no desenvolvimento que a encoprese pode trazer, considerase importante realizar um levantamento de dados científicos para avaliação e análise da produção científica sobre o assunto na literatura brasileira (Baptista \& Campos, 2007). Desse modo, esse artigo propõe uma revisão que objetiva mapear, apontar o referencial teórico e suas diferenças em artigos publicados sobre intervenções psicológicas em encoprese.

\section{Método}

\subsection{Procedimento}

Para a seleção dos artigos foram consideradas as obras nacionais encontradas nas bases de dados Scielo e Lilacs, nos últimos 20 anos, no período de 1994 a 2013, por conveniência das pesquisadoras devido à falta de digitalização dos artigos antes dessa data, categorizados em relatos e estudo de caso, de forma a abranger a literatura nacional que apresenta estratégias de intervenção para a encoprese. Os descritores utilizados para a busca foram: encoprese, transtorno de evacuação, incontinência fecal e constipação intestinal. Também foi utilizado como critério de escolha os artigos cujo texto completo estava disponível nas bases de dados. Após a seleção dos textos, os dados foram analisados e categorizados tendo em vista: a) o procedimento adotado para o tratamento da encoprese, b) os resultados, c) o referencial teórico, d) comparar e confrontar as posições sobre o assunto, e) identificar as lacunas de conhecimento em relação ao tema e f) constatar os resultados e as medidas de precisão (Baptista \& Campos, 2007; Luna, 2005).

\section{Resultados}

Após as buscas, foi encontrado um total de 231 artigos. Por meio da leitura dos resumos, os artigos que não eram da área da psicologia 
ou que não apresentavam relato de intervenção foram excluídos da amostra. Desse modo, apenas cinco artigos caracterizados como estudo de caso foram selecionados.

Na Figura 1 estão descritos os critérios e os passos realizados para a seleção dos artigos encontrados nas bases de dados Scielo e Lilacs.

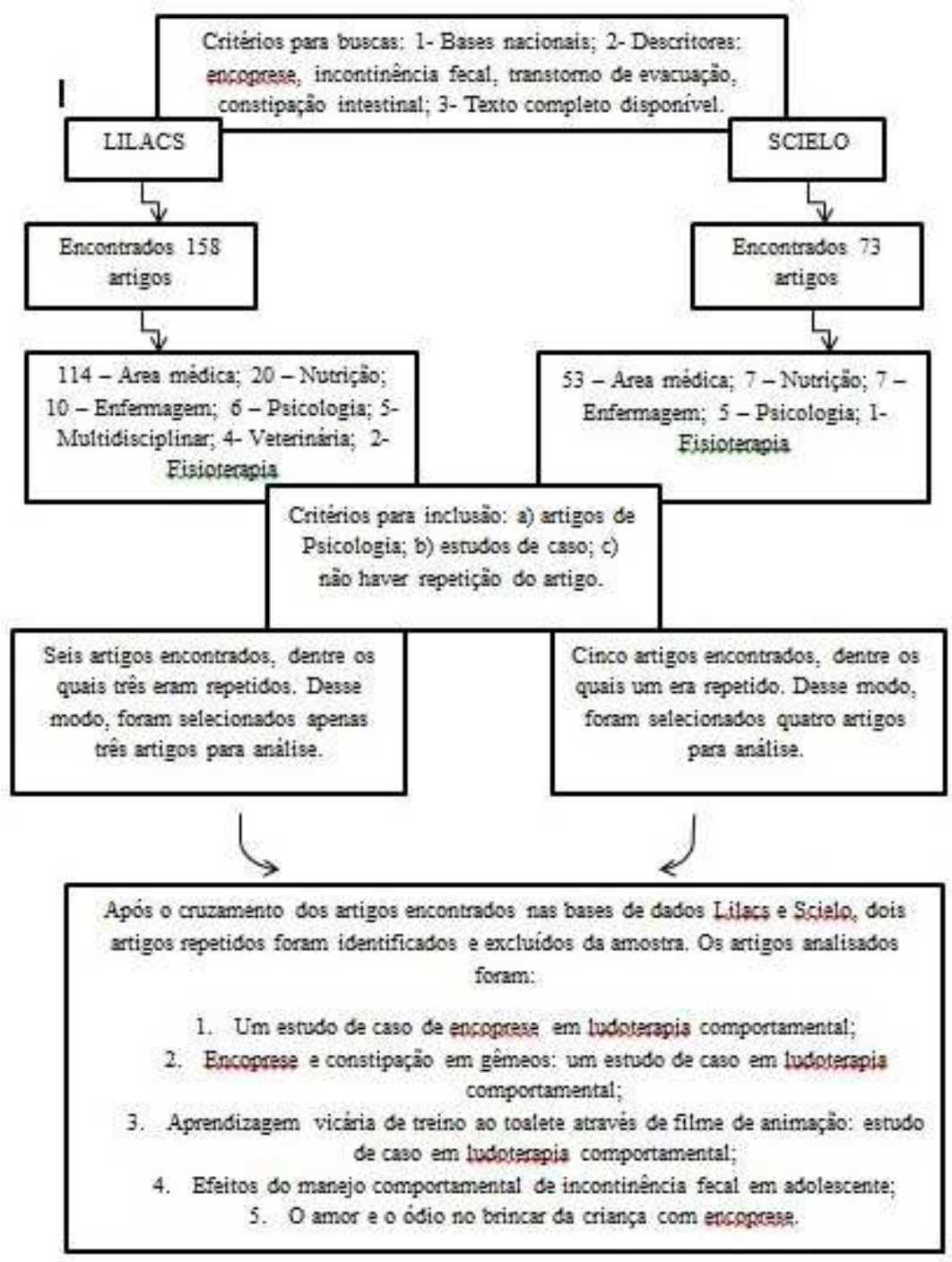

Figura 1. Passos e critérios para a seleção de artigos nas bases de dados utilizadas.

A Tabela 1 apresenta as características gerais e referenciais teóricos dos trabalhos analisados a partir da data de publicação.

Tabela 1. Características gerais e referenciais teóricos dos artigos analisados 


\begin{tabular}{|c|c|c|c|c|}
\hline Autores & Ano & Revista & $\begin{array}{l}\text { Referencial } \\
\text { Teórico }\end{array}$ & Título \\
\hline $\begin{array}{l}\text { Gomes, } \\
\text { L. S. }\end{array}$ & 1998 & $\begin{array}{l}\text { Psicologia: } \\
\text { Ciência e } \\
\text { Profissão }\end{array}$ & $\begin{array}{c}\text { Análise do } \\
\text { Comportamento }\end{array}$ & $\begin{array}{l}\text { Um estudo de } \\
\text { caso de } \\
\text { encoprese em } \\
\text { ludoterapia } \\
\text { comportamental }\end{array}$ \\
\hline $\begin{array}{l}\text { Coelho, } \\
\text { L. S. G. }\end{array}$ & 2001 & $\begin{array}{c}\text { Psicologia: } \\
\text { Ciência e } \\
\text { Profissão }\end{array}$ & $\begin{array}{l}\text { Análise do } \\
\text { comportamento }\end{array}$ & $\begin{array}{l}\text { Encoprese e } \\
\text { constipação em } \\
\text { gêmeos: um } \\
\text { estudo de caso } \\
\text { em ludoterapia } \\
\text { comportamental }\end{array}$ \\
\hline $\begin{array}{l}\text { Coelho, } \\
\text { L. S. G. }\end{array}$ & 2008 & $\begin{array}{l}\text { Psicologia: } \\
\text { Ciência e } \\
\text { Profissão }\end{array}$ & $\begin{array}{c}\text { Análise do } \\
\text { Comportamento }\end{array}$ & $\begin{array}{c}\text { Aprendizagem } \\
\text { vicária de treino } \\
\text { de toalete } \\
\text { através de filme } \\
\text { de animação: } \\
\text { estudo de caso } \\
\text { em ludoterapia } \\
\text { comportamental }\end{array}$ \\
\hline $\begin{array}{l}\text { Barbieri, } \\
\text { V., } \\
\text { Ujikawa, } \\
\text { M. I. \& } \\
\text { Mishima, } \\
\text { F. K. T. }\end{array}$ & 2011 & $\begin{array}{c}\text { Tempo } \\
\text { Psicanalítico }\end{array}$ & Psicanálise & $\begin{array}{l}\text { O amor e o ódio } \\
\text { no brincar da } \\
\text { criança com } \\
\text { encoprese }\end{array}$ \\
\hline $\begin{array}{l}\text { Neves, } \\
\text { A. J. \& } \\
\text { Calais, } \\
\text { S. L. }\end{array}$ & 2012 & $\begin{array}{l}\text { Psicologia: } \\
\text { Ciência e } \\
\text { Profissão }\end{array}$ & $\begin{array}{c}\text { Análise do } \\
\text { Comportamento }\end{array}$ & $\begin{array}{c}\text { Efeitos do } \\
\text { manejo } \\
\text { comportamental } \\
\text { de incontinência } \\
\text { fecal em } \\
\text { adolescente }\end{array}$ \\
\hline
\end{tabular}

A partir da leitura dos trabalhos selecionados, foi possível concluir que dentre os cinco artigos, três foram escritos pela mesma autora (Coelho, L. S. G, 2001; Coelho, L.S.G., 2008; Gomes, L.S, 1998). Esse dado indica que além de haver escassos artigos nacionais publicados sobre encoprese, ainda existem poucos autores publicando sobre o tema. Também há predomínio da revista de publicação, sendo que a "Psicologia: Ciência e Profissão", revista com Qualis A2 do Conselho Federal de Psicologia, contém quatro dos cinco artigos publicados sobre o transtorno em psicologia nos últimos 20 anos. Ainda, as intervenções realizadas são predominantemente no âmbito 
da análise do comportamento $(n=4)$, e apenas um na abordagem psicanalítica. Em relação ao público alvo dos trabalhos, foram sete crianças e um adolescente, com idade entre três e 14 anos, destes sete eram meninos e apenas uma menina. Assim, as intervenções foram realizadas predominantemente com o sexo masculino, corroborando os dados apontados sobre frequência masculina do transtorno no estudo de Wendy e William (2006).

Pode-se observar que nos quatro artigos publicados na abordagem comportamental, os autores relataram os procedimentos de avaliação, intervenção e resultados obtidos. As avaliações, de modo geral, consistiram em entrevistar os pais, responsáveis e cuidadores; investigar a história de vida do cliente, o histórico da queixa encoprética, a alimentação, a vida escolar, o relacionamento social, a saúde e os tratamentos anteriores; solicitar registo da ocorrência (frequência e intensidade) do comportamento alvo; realizar sessões com os clientes e familiares e efetuar a análise funcional.

Tratando-se das intervenções, em relação ao número de atendimentos houve variações, mas a sequência dos procedimentos utilizados foram semelhantes nos estudos: estabelecer linha de base; favorecer o vínculo terapêutico; habituar o comportamento de encoprese por meio da diminuição de respostas fisiológicas relacionadas à defecação e dessensibilização ao vaso sanitário; reforçar positivamente os comportamentos de autonomia e assertividade; orientar os responsáveis sobre estratégias de treino e o que mantém o comportamento de incontinência fecal; propor situações de defecação por via lúdica; reduzir uso de medicação e o intervalo entre as defecações; alterar o padrão de alimentação; treinar o controle da defecação e o uso ao toalete; favorecer a interação familiar; modelar o relato do cliente sobre a encoprese; ensinar análise funcional ao cliente; realizar fading out e redefinir auto regra de limpeza e sujeira; ensinar o cliente a registrar os comportamentos de encoprese e monitoramento; estabelecer rotina, horários e tarefas e avaliar o desempenho do cliente.

Quanto aos resultados, os quatro estudos obtiveram êxito ao demonstrarem a remissão dos comportamentos encopréticos, a retirada ou redução no uso da medicação, diminuição do tempo de intervalo entre as defecações, padrão fixo de uso à toalete, controle de esfíncter e aprendizagem de comportamentos de defecação adequados. Ainda, todos os pesquisadores analistas do comportamento fizeram follow-up, e verificaram que as aprendizagens haviam se mantido.

Enquanto isso, o artigo com referencial psicanalítico descreveu a avaliação e a análise realizadas a partir do relato da família de filhos com queixas encopréticas. O procedimento de intervenção, realizado com três crianças, constou de entrevistas com os pais, sessões lúdicas com as crianças e análise psicanalítica da encoprese, ou seja, 
a interpretação dos sintomas por meio dos pressupostos da psicanálise. Foi discutido que a rigidez materna, associada à dificuldade em integrar os impulsos destrutivos e amorosos, não auxilia a criança na aquisição das capacidades de simbolização, o que reflete no desenvolvimento do transtorno. Apesar de encontrar aspectos da análise comum aos três casos, o artigo descreve de forma pouco pormenorizada o processo psicoterápico pela qual as crianças passaram, dificultando a identificação do procedimento adotado para a remissão do quadro encoprético. As autoras apontaram para a necessidade de um atendimento clínico para a criança com encoprese e para sua família, a fim de garantir um ambiente que favoreça o desenvolvimento saudável da criança, assim como a importância do contato da família com o analista que recebe e nomeia as experiências emocionais da criança e dos pais.

\section{Discussão}

Conforme apontado anteriormente por Neves e Calais (2012), foi possível constatar que há um número restrito de estudos no Brasil sobre o tema encoprese na área da Psicologia, além da maior frequência no referencial comportamental e em uma mesma revista. Parece que a medicina é quem mais se preocupa com o tema, talvez porque os responsáveis pelas crianças/adolescentes que apresentam tais dificuldades encarem esta questão como fisiológica e sem relação com o comportamento.

Dentre as publicações selecionadas para a análise neste estudo, as contribuições da análise do comportamento foram consideradas importantes, por descreverem os procedimentos utilizados previamente à intervenção, a intervenção em si e os resultados obtidos; e ainda a partir do follow-up realizado em todas as pesquisas, demonstrou que os comportamentos aprendidos se mantiveram e o quadro encoprético continuou reduzido ou extinto do repertório dos clientes, demonstrando a eficácia desses procedimentos. Como a encoprese é um transtorno cujas determinações advêm de muitas variáveis, é importante considerar que, tanto o processo de avaliação quanto os procedimentos adotados para a remissão do quadro, consideraram esse aspecto. Assim, para a avaliação do caso foram apreciadas a relação familiar e escolar, a história de vida, com destaque para o período do desfralde, alimentação, rotina. De forma semelhante, por meio do manejo terapêutico, foram investigados todos os aspectos da vida que pareceram estar relacionados ao transtorno.

Os procedimentos adotados justificam-se na medida em que a análise do comportamento compreende os comportamentos típicos do quadro de encoprese como derivados de aprendizagens operantes e não 
apenas como respostas inatas do organismo (Mota, Barros, Matijasevich, \& Santos, 2010). Por isso, é importante que se realize uma vasta investigação comportamental, que possibilite a compreensão de quais contingências controlam as respostas relacionadas ao transtorno, sua função no ambiente e as reservas comportamentais que auxiliam terapeuta e cliente durante o processo terapêutico (Coelho, 2008; Silvares \& Souza, 2001). Identificadas tais contingências, os procedimentos adotados têm em vista extinguir os comportamentos relacionados à encoprese e proporcionar o desenvolvimento de outros repertórios comportamentais, socialmente mais valorizados (Brooks, Copen, Cox, Morris, Borowitz, \& Stupen, 2000).

Assim, é possível afirmar que nos artigos encontrados, segundo a abordagem analítico comportamental, foram utilizadas estratégias que a literatura aponta como eficazes para a remissão do quadro relativo à encoprese, como: modelagem comportamental (Coehlo, 2011; Magee, Ritterband, Thorndike, Cox, \& Borowitz, 2009); reforçamento diferencial de comportamentos distintos dos tipicamente identificados para o transtorno (Edelman, 1971); estabelecimento de contingências de reforçamento com o intuito de viabilizar o desenvolvimento de operantes, para uso regular do toalete, a discriminação de reações interoceptivas (Cox, Stuphen, Borowitz, Kovatchev, \& Ling, 1998) e orientação para os pais. Além disso, ressalta-se a importância do estabelecimento do vínculo terapêutico, a fim de que se estabeleça um contexto não coercitivo que possibilite que o cliente discorra sobre os eventos relacionados à encoprese e diminua a incidência de comportamentos de esquiva (Conger,1970).

Com relação ao artigo psicanalítico, notou-se que foram descritas as etapas de avaliação dos participantes, mas não ficaram explícitos os processos de intervenção ou possíveis resultados, apontando a necessidade de um atendimento clínico para a criança com encoprese e sua família, a importância da experiência com o analista, a influência da relação com a mãe para o processo de simbolização da criança. Contudo, o artigo não fornece dados empíricos de que seguindo essas orientações o procedimento será efetivo. É possível inferir que a maneira como os dados foram apresentados e discutidos se adequam à ótica da abordagem sobre o fenômeno da encoprese e sobre a fase de desfralde.

Ana Freud (1971/1987) afirma que a aquisição do controle esfincteriano pela criança inclui aspectos como a sua liberdade para defecar e urinar e a interferência materna nesse processo. Quando isso ocorre, o impulso libidinal da criança é transferido da zona oral para a anal, assim seus produtos corporais ganham grande investimento libidinal, acarretando ambivalência do mundo objetal e 
transferindo a preocupação com a limpeza e sujidade do objeto externo para o ego e superego da criança.

Então, durante o tratamento psicoterapêutico, procura-se por meio da transferência, vivenciar a relação de amor e ódio com relação às figuras parentais, viabilizando, com o processo de simbolização, a desobrigação da criança a atuar diretamente com as pulsões do ambiente. Por isso, considera-se importante investigar como no decorrer do processo psicoterápico a criança utiliza o espaço potencial bem como a maneira como a ambivalência se manifesta (Winnicott, 1993).

Diante do exposto acima, é importante refletir sobre a ausência de publicação sobre assunto tão relevante. Pode-se considerar que a falta de publicações talvez seja devida às dificuldades apresentadas por familiares e pelas crianças em buscar ajuda e assumir a condição do transtorno, uma vez que a ajuda pode ser adiada por prevalência de sentimentos como vergonha e culpa (Morais \& Fernandes, 2011).

As técnicas utilizadas pelos pesquisadores tiveram êxito, todavia foram realizadas com amostras pequenas devido ao tipo de estudo. Seria necessário que as mesmas técnicas e procedimentos fossem utilizados em amostra maior, com outro desenho metodológico, para dar maior validade e confiabilidade ao modelo comportamental.

\section{Referências}

American Psychiatric Association (2002). Diagnostic and statistical manual of mental disorders fourth edition revised (DSM-IVR). Washington, DC: APA.

Baptista, M. N., \& Campos, D. C. (2007). Metodologias de pesquisa em ciências: análise quantitativa e qualitativa. Rio de Janeiro: LTC.

Barbieri, V., Ujikawa, M. I., \& Mishima, F. K. T. (2011). O amor e o ódio no brincar da criança com encoprese. Tempo psicanalítico, 43(2), 321-337.

Blum, N. J., Taubman, B., \& Nemeth, N. (2004). During toilet training, constipation occurs before stool toileting refusal. Pediatrics, 113, 1809-1810.

Bragado, C. A. (1998). Encopresis. Madri: Editora Pirâmide.

Brazelton, T. B. (1962). A child-oriented approach to toilet training. Pediatrics, 29(1), 121-128.

Brooks, R. C., Copen, R. M., Cox, D. J., Morris, J., Borowitz, S., \& Sutphen, J. (2000). Review of the treatment literature for encopresis, functional constipation, and stool-toileting refusal. Annals of Behavioral Medicine, 22(3), 260-267.

Coehlo, D. P. (2011). Encopresis: A medical and family approach. Pediatric Nursing, 37(3), 107-112. 
Coelho, L. S. G. (2001). Encoprese e constipação em gêmeos: um estudo de caso em ludoterapia comportamental. Psicologia: Ciência e profissão, 21(1), 2-13.

Coelho, L. S. G. (2008). Aprendizagem vicária de treino de toalete através de filme de animação: estudo de caso em ludoterapia comportamental. Psicologia: Ciência e profissão, 28(4), 846861.

Conger, J. C. (1970). The treatment of encopresis by the management of social consequences. Behavior Therapy, 1(3), 386-390.

Cox, D. J., Sutphen, J., Borowitz, S., Kovatchev, B., \& Ling, W. (1998). Contribution of behavior therapy and biofeedback to laxative therapy in the treatment of pediatric encopresis. Annals of Behavioral Medicine, 20(2), 70-76.

Cox, D. J., Sutphen, J., Ling, W., Quillian, W., \& Borowitz, S. (1996). Additive benefits of laxative, toilet training and biofeedback therapies in the treatment of pediatric encopresis. Journal of pediatric psychology, 21(5), 659-670.

Cunningham, C. L., \& Banez, G. A. (2006). Defecation disorders. In C. L. Cunningham, \& G. A. G. Banez (Orgs.). Pediatric gastrointestinal: disorder biopsychsocial assessment and treatment, (pp.127-160). New York: Springer.

Edelman, R. I. (1971). Operant conditioning treatment of encopresis. Journal Behavior Therapy \& Experimental Psychiatry, 2(1), 7173.

Fleisher, D. R. (2004). Understanding toilet training difficulties. Pediatrics, 113(6), 1809-1810.

Freud, A. (1971/1987). Do "molhar e sujar" as fraldas ao controle da bexiga e dos intestinos. In A. Freud (Org.). Infância normal e patológica: determinantes do desenvolvimento, ( $\mathrm{pp} .68-70)$. Rio de Janeiro: Zahar.

Gomes, L. S. (1998). Um estudo de caso de encoprese em ludoterapia comportamental. Psicologia: Ciência e Profissão, 18(3), 54-61.

Knell, S. M. (1995). Cognitive behavioral play therapy. New Jersey: Aronson.

Luna, S. V. (2005). Planejamento de pesquisa: uma introdução. Elementos para uma análise metodológica. São Paulo: Educ.

Magee, J. C., Ritterband, L. M., Thorndike, F. P., Cox, D. J., \& Borowitz, S. M. (2009). Exploring the relationship between parental worry about their children's health and usage of an internet intervention for pediatric encopresis. Journal of Pediatric Psychology, 34(5), 530-538.

Morais, M. C., \& Fernandes, J. L. (2011). Abordagem da encoprese na criança. Revista saúde infantil, 33 (2), 58-64. 
Mota, D. M., Barros, A. J., Matijasevich, A., \& Santos, I. S. (2010). Avaliação longitudinal do controle esfincteriano em uma coorte de crianças brasileiras. Jornal de Pediatria, 86(5), 429-434.

Neves, A. J., \& Calais, S. L. (2012). Efeitos do manejo comportamental de incontinência fecal em adolescente. Psicologia: Ciência e profissão, 3(32), 754-767.

Pinheiro, H. B., \& Moreno, M. (1983). Encoprese - um enfoque clínico, diagnóstico e terapêutico. Revista Brasileira Coloproctologia, 3(1), 19-24.

Silvares, E. F. M. (2001). Ludoterapia cognitivo-comportamental com crianças agressivas. In H. J. Guilhardi, M. B. B. P. Madi, P. P. Queiroz, \& M. C. Scoz (Orgs). Sobre comportamento e cognição: expondo a variabilidade, Santo André: Esetec, 71-90.

Silvares, E. F. M. \& Souza, C. L. (2001). Prevenção e tratamento comportamental dos problemas de eliminação na infância. Temas em Psicologia, 9(2), 99-111.

Wendy, B., \& William, D. (2006). Evaluation and treatment of constipation in infants and children. American Family Physician, 73: 469-77.

Winnicott, D. W. (1979/1993). O ambiente e os processos de maturação. Porto Alegre: Artes Médicas.

\section{Endereço para correspondência \\ Jéssica Aline Rovaris}

Universidade Estadual Paulista - UNESP

Programa de pós-graduação em Psicologia do Desenvolvimento e Aprendizagem

Av. Luiz Edmundo Carrijo Coube, 14-01, CEP 17033-360, Bauru - SP, Brasil

Endereço eletrônico: jessica.rovaris@gmail.com

\section{Bárbara Trevizan Guerra}

Universidade Estadual Paulista - UNESP

Programa de pós-graduação em Psicologia do Desenvolvimento e Aprendizagem

Av. Luiz Edmundo Carrijo Coube, 14-01, CEP 17033-360, Bauru - SP, Brasil

Endereço eletrônico: barbaratrevizan@hotmail.com

\section{Sandra Leal Calais}

Universidade Estadual Paulista - UNESP

Programa de pós-graduação em Psicologia do Desenvolvimento e Aprendizagem

Av. Luiz Edmundo Carrijo Coube, 14-01, CEP 17033-360, Bauru - SP, Brasil

Endereço eletrônico: scalais@fc.unesp.br

\section{Carmen Maria Bueno Neme}

Universidade Estadual Paulista - UNESP

Programa de pós-graduação em Psicologia do Desenvolvimento e Aprendizagem

Av. Luiz Edmundo Carrijo Coube, 14-01, CEP 17033-360, Bauru - SP, Brasil

Endereço eletrônico: cmneme@gmail.com

Recebido em: 30/05/2014

Reformulado em: 18/11/2014

Aceito para publicação em: 01/12/2014

\section{Notas}


* Psicóloga e mestranda do programa de pós-graduação em Psicologia do Desenvolvimento e Aprendizagem - Unesp Bauru.

** Psicóloga e mestranda do programa de pós-graduação Psicologia do Desenvolvimento e Aprendizagem - Unesp Bauru

*** Psicóloga, doutora em Psicologia e docente do programa de pós-graduação em Psicologia do Desenvolvimento e Aprendizagem da Universidade Estadual Paulista UNESP/Bauru.

**** Psicóloga, livre-docente e docente do programa de pós-graduação em Psicologia do Desenvolvimento e Aprendizagem da Universidade Estadual Paulista UNESP/Bauru. 